\title{
Plastic deformation of decagonal Al-Ni-Co quasicrystals
}

\author{
By M. Feuerbacherł, M Bartschł, B, Grushro $\dagger$, \\ U. Messerschmidt $\$$ and K. Urban $† \S$ \\ $\dagger$ Institut für Festkörperforschung, Forschungszentrum Jülich GmbH, \\ D-52425 Jülich, Germany \\ ¥ Max-Planck-Institut für Mikrostrukturphysik, D-06120 Halle, Germany
}

[Received 8 August 1997 and accepted 17 September 1997]

\begin{abstract}
Plastic deformation experiments have been performed on Czochralski-grown decagonal Al-Ni-Co single-quasicrystals at temperatures between 780 and $860^{\circ} \mathrm{C}$. Compression tests at a strain rate of $10^{-5} \mathrm{~s}^{-1}$ with different orientations of the compression axis relative to the tenfold quasicrystal direction show an anisotropy of the plastic behaviour. If the compression axis is oriented parallel to the tenfold direction multiple slip and weak work hardening is observed. If the compression axis is tilted by $45^{\circ}$, single-slip conditions and deformation softening are found. Microstructural investigation by transmission electron microscopy indicates that plastic deformation is mediated by a dislocation mechanism. The results are interpreted in terms of a model in which the chemically ordered columnar clusters of the decagonal structure represent rate-controlling obstacles.
\end{abstract}

\section{§ 1. Introduction}

The mechanical properties of quasicrystalline materials have been the subject of numerous investigations in recent years. It has been found that the plastic behaviour of icosahedral quasicrystals exhibits special features not observed in conventional metallic alloys. The most remarkable property is the absence of work hardening, first observed in experiments on polyquasicrystalline materials (Takeuchi et al. 1991, Kang and Dubois 1992, Bresson and Gratias 1993). Experiments on single-quasicrystalline icosahedral Al-Pd-Mn not only confirmed this behaviour but also demonstrated that plasticity is mediated by a dislocation mechanism (Wollgarten et al. 1993, 1995). Quantitative experiments on the same material permitted measurements of the thermodynamic activation parameters for plastic deformation (Feuerbacher et al. 1995). These results provided the basis of a model for quasicrystal plasticity suggested recently by Feuerbacher et al. (1997) in which a key part is played by the cluster substructure of icosahedral quasicrystals.

In decagonal quasicrystals, the atoms are arranged in a lattice which is quasiperiodic in two dimensions and periodic along the third. With respect to the axis perpendicular to the periodically stacked quasiperiodic planes, diffraction patterns exhibit a tenfold rotational symmetry (for a review see Steurer (1996)). It was found that the structural anisotropy induces corresponding anisotropies in the physical properties. In particular, this holds for the electronic properties (for example, Lin et al. 1990, Basov et al. 1994). In contrast, Vickers hardness measurements on

$\S$ On sabbatical leave at: Institute for Advanced Materials Processing, Tohoku University, Sendai, Japan. 
single-quasicrystalline decagonal Al-Cu-Co-Si by Wittmann et al. (1991) did not show any anisotropy.

Since, until now, larger single-quasicrystals of decagonal phases could not be produced, mechanical-property studies on decagonal quasicrystals are rare and, with the already mentioned exception, they have been performed on polyquasicrystalline material (Köster et al. 1993, Mishra et al. 1993, Wang et al. 1993). We recently succeeded in growing centimetre-sized grains of decagonal Al-Ni-Co oriented along the tenfold axis (B. Grushko, M. Beyss and K. Fischer 1997, unpublished). In the present letter we report on the first quantitative plastic deformation tests carried out on this material.

\section{§ 2. EXPERIMENTAL}

\subsection{Quasicrystal growth}

The quasicrystal was grown under vacuum by means of the Czochralski technique from a melt of composition Al-75 at $\%, \mathrm{Ni}-14.5 \mathrm{at} \%, \mathrm{Co}-10.5 \mathrm{at} \%$ which was induction heated in an alumina crucible. The crucible and the seed were both rotated at $20 \mathrm{rpm}$ in opposite directions in order to achieve optimum mixing of the melt. The pulling speed was adjusted to $7 \mathrm{~mm} \mathrm{~h}^{-1}$. The pulling direction, as defined by the orientation of the seed, was parallel to the direction of the tenfold quasicrystal axis (henceforth abbreviated by $A 10$ ). The single-quasicrystalline seed was extracted from an ingot which was melted, slowly cooled and subsequently subjected to a suitable heat treatment. By pulling carefully a very thin neck at the beginning of growth, single-grain growth conditions can be achieved. Nevertheless, owing to a particularly low nucleation threshold for secondary grains in low-angle orientation, the resulting quasicrystal of about $30 \mathrm{~mm}$ in length and $8 \mathrm{~mm}$ in diameter consists of typically three to five columns of tenfold orientation with misorientation angles of less than $2^{\circ}$ and relative rotation angles around the tenfold axis of less than $10^{\circ}$.

The quasicrystal used for the present work consisted of four parallel grains. Its composition, as determined by inductively coupled plasma optical emission spectroscopy, was $\mathrm{Al}_{71 \cdot 7} \mathrm{Ni}_{15 \cdot 4} \mathrm{Co}_{12 \cdot 9}$. Material of this and neighbouring compositions was extensively studied by Grushko and Urban (1994). X-ray diffractometry, optical microscopy, scanning electron microscopy and transmission electron microscopy in longitudinal as well as in transverse cross-sectional cuts showed that the quasicrystal consisted entirely of the decagonal phase. No secondary phases could be detected.

\subsection{Sample preparation and treatments}

For the deformation experiments samples of $1.5 \mathrm{~mm} \times 1.5 \mathrm{~mm} \times 5 \mathrm{~mm}$ were cut from the quasicrystal. Owing to their smallness, they contained only one grain boundary at the most. For a first set of samples, the long axis was oriented parallel to the tenfold quasicrystal lattice direction. In a second set, the long axis was tilted by $45^{\circ}$ with respect the tenfold direction. All samples were carefully ground and polished in order to prevent crack formation at the surfaces. The plastic deformation experiments were carried out in compression along the long sample axis in air at temperatures between 780 and $860^{\circ} \mathrm{C}$. An Instron 8562 testing system was used under closed loop control at a constant strain rate of $10^{-5} \mathrm{~s}^{-1}$. 
From the stress decay rate $\dot{\sigma}$ measured during stress-relaxation experiments, the strain-rate dependence of the flow stress $I$ and the activation volume $V$ were determined according to the relation

$$
V=\frac{k T}{m_{\mathrm{s}} I}=\left.\frac{k T}{m_{\mathrm{s}}} \frac{\partial \ln (-\dot{\sigma})}{\partial \sigma}\right|_{T},
$$

where $\sigma$ is the applied stress, $m_{\mathrm{s}}$ is the Schmid factor, $k$ is Boltzmann's constant and $T$ is the absolute temperature (for example, Kocks et al. (1975)). The stress exponent $m$ was determined using

$$
m=\left.\frac{\partial \ln (-\dot{\sigma})}{\partial \ln (\sigma)}\right|_{T} .
$$

After deformation, the samples were rapidly unloaded and cooled at a rate of about $12^{\circ} \mathrm{C} \mathrm{min}^{-1}$. The surfaces of the samples were inspected by phase contrast optical microscopy. Microstructural investigations of the deformed samples and of undeformed reference samples were carried out in a JEOL 2000EX transmission electron microscope operated at $200 \mathrm{kV}$.

\section{§ 3. Results}

Figure $1(a)$ shows the true stress as a function of true strain for samples compressed along $A 10$ at 780 and $815^{\circ} \mathrm{C}$. During the deformation at the lower temperature, two stress relaxations were carried out which resulted in corresponding transient stress drops (R1, R2). A very broad onset of plastic yielding is observed. At $780^{\circ} \mathrm{C}$ the stress reaches a maximum value of $200 \mathrm{MPa}$ at $1.3 \%$ of strain. This is followed by a yield drop by about $2 \%$ after which the stress rises again. At $815^{\circ} \mathrm{C}$ the stress increases to a plateau value of $125 \mathrm{MPa}$ at $1.3 \%$ strain. Starting from about $3 \%$ strain a further stress increase is observed.

Figure $1(b)$ shows the corresponding stress-strain curves for samples whose compression axis was tilted by $45^{\circ}$ with respect to $A 10$. At both temperatures a
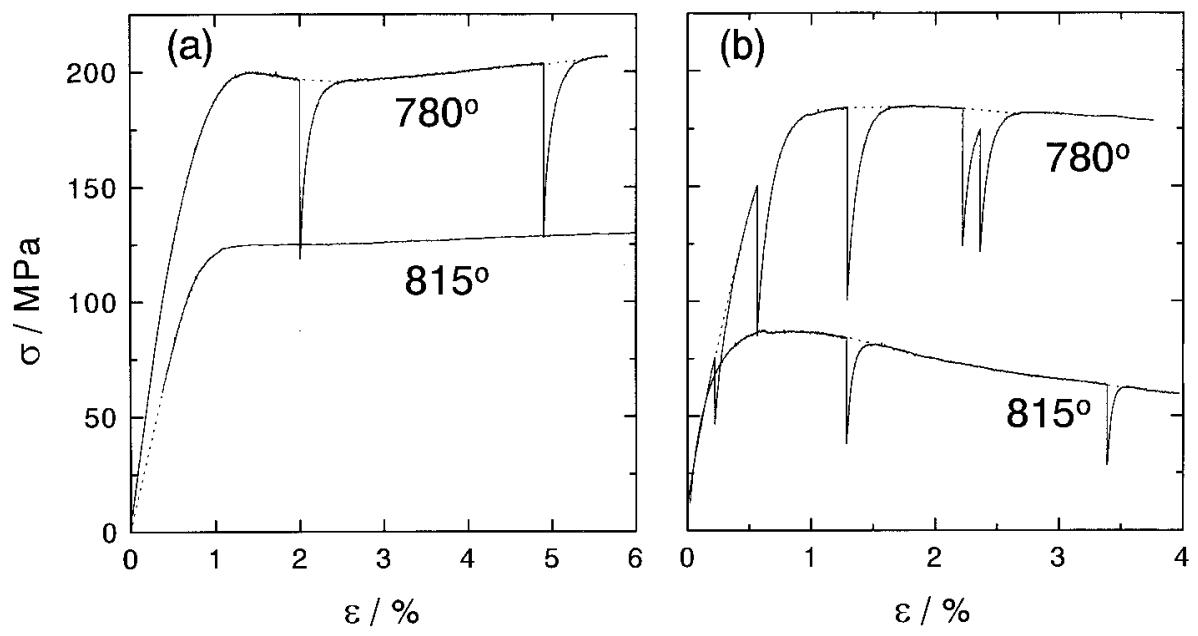

Figure 1. True stress against true strain for $\mathrm{Al}_{71.7} \mathrm{Ni}_{15.4} \mathrm{Co}_{12.9}$ decagonal quasicrystal deformed at a rate of $10^{-5} \mathrm{~s}^{-1}$. (a) Compression axis parallel to the tenfold quasicrystal axis and $(b)$ along an axis tilted by $45^{\circ}$ with respect to the tenfold quasicrystal axis. 
number of stress relaxation experiments were carried out. Again a rather broad onset of plastic yielding is observed. The maximum stress is smaller than in the case of compression along $A 10$, that is $183 \mathrm{MPa}$ at $780^{\circ} \mathrm{C}$ and $87 \mathrm{MPa}$ at $815^{\circ} \mathrm{C}$. After this is reached, the stress continuously decreases. The temperature dependence of the maximum stress (after yielding) is given in figure 2 for both specimen orientations.

The strain-rate dependence of the flow stress $I$ rises linearly with stress from $2.3 \mathrm{MPa}$ at $20 \mathrm{MPa}$ to $34 \mathrm{MPa}$ at $200 \mathrm{MPa}$. For the evaluation of the activation volume $V$ according to equation (1) we have assumed a tentative value for the Schmid factor of $m_{\mathrm{s}}=0.5$ for both orientations. We find values between 14 and $1 \mathrm{~nm}^{3}$ at 20 and $200 \mathrm{MPa}$ respectively. Figure 3 shows the stress dependence of $V$. We find a hyperbolic behaviour which does not depend on sample orientation. For the stress exponent $m$ we find values between $4 \cdot 5$ and 5.5.

On the surface of the deformed samples, light microscopy showed a high density of slip lines. In the case of the samples compressed parallel to $A 10$ these lines formed a rather irregular pattern. In the case of the samples deformed along an axis tilted by $45^{\circ}$, only a single set of parallel lines with a spacing of the order of $0.1 \mathrm{~mm}$ was observed. On two opposite side faces of the sample, these lines were found to be tilted by $45^{\circ}$ with respect to the compression axis, marking the intersection of the specimen surfaces and the quasiperiodic planes (by this expression we denote the planes whose normal is parallel to $A 10$ ).

Under two-beam bright-field conditions in the transmission electron microscope, the deformed samples show a high density of inhomogeneously distributed dislocations. Two types of dislocations can be observed. The majority consists of parallely aligned straight dislocations. Only a few curved dislocations are found. At $815^{\circ} \mathrm{C}$, the total dislocation density amounts to about $4 \times 10^{9} \mathrm{~cm}^{-2}$ in the high density and $10^{7} \mathrm{~cm}$ in the low density areas. Undeformed reference samples subjected to the same heat treatment as the deformed ones contain a significantly lower density of dislocations, of the order of the value found in the low density areas in the deformed specimens. Contrast extinction experiments gave the following results on the Burgers

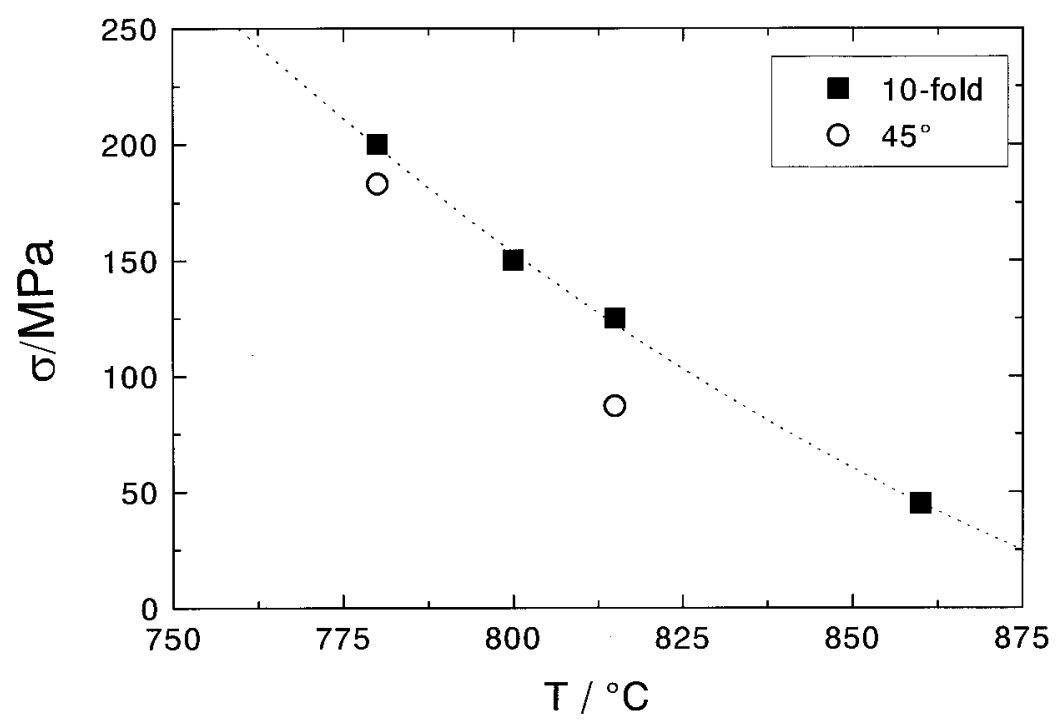

Figure 2. Temperature dependence of the maximum stress for different specimen orientations. 


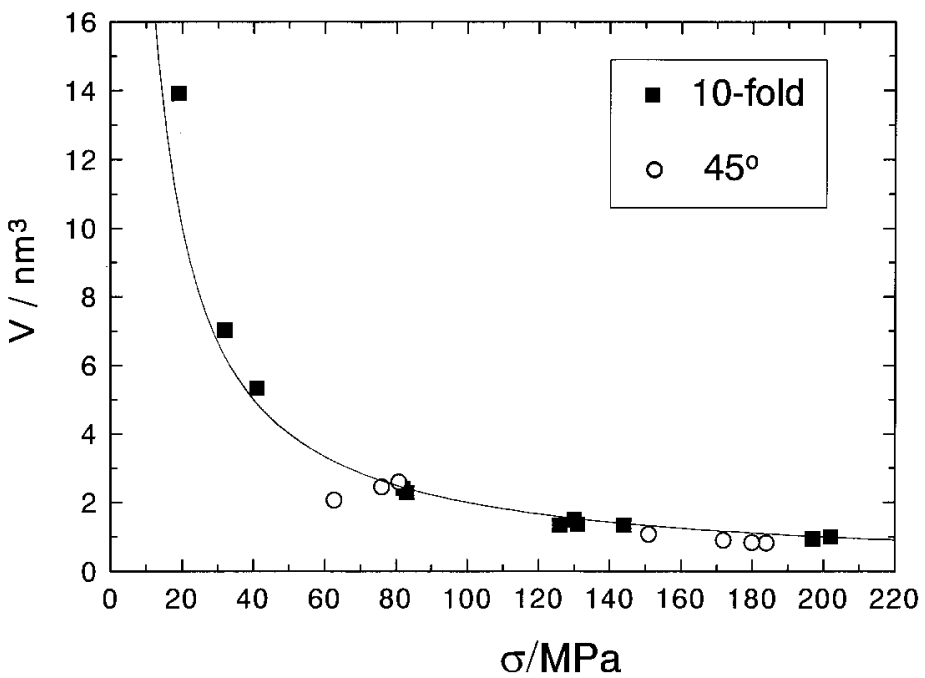

Figure 3. Stress dependence of the activation volume $V$ at the two specimen orientations.

vector directions of dislocations in the deformed material. In the samples with the compression axis tilted by $45^{\circ}$ with respect to $A 10$, the major proportion of the dislocations, that is the aligned and straight ones, have Burgers vectors lying in the quasiperiodic planes. In the samples deformed along $A 10$, the major proportion of the dislocations possess Burgers vectors which are inclined to both the compression direction and $A 10$.

\section{$\S 4$. Discussion}

Our observation of a strong increase in dislocation density in deformed samples, by more than two orders of magnitude at $815^{\circ} \mathrm{C}$, provides direct evidence for the fact that, as in icosahedral quasicrystals, plastic flow in decagonal quasicrystals is mediated by a dislocation mechanism.

In our experiments we observe clear differences depending on whether the samples are deformed along the tenfold quasicrystal axis or parallel to a direction making an angle of $45^{\circ}$ with this axis. In the first case, after a small yield drop, the stress rises with increasing strain. The samples show a pattern of entangled slip lines on the surface, and the Burgers vectors of the deformation-induced dislocations are inclined with respect to the quasiperiodic planes. In the second case, after a maximum, the stress decreases continuously with strain. Here the slip line pattern consists of a single set of parallel lines and the dislocation Burgers vectors lie in the quasiperiodic planes.

For a discussion, we first consider the possible slip systems. According to Schmid's law (for example Courtney (1990)), the maximum resolved shear stress occurs in planes tilted with respect to the compression axis by an angle of $45^{\circ}$. Yan et al. (1993) have carried out a detailed convergent-beam electron-diff raction analysis on the quasicrystal axes of decagonal Al-Ni-Co. From their work we find that, for the case of the compression axis parallel to $A 10$, there are a number of lowindex planes which can be considered good candidates for slip planes. In particular, this holds for the planes having the $F$ and $J^{\prime}$ axes as plane normals making an angle of $46^{\circ}$ and $40^{\circ}$ respectively with $A 10$. However, since, according to the tenfold 
rotational symmetry, ten of each of these axes are arranged around the compression axis, multiple rather than single slip is expected to occur right from the onset of plastic deformation during compression along $A 10$. Evidence for this is provided by the very irregular pattern of entangled slip lines on the specimen surfaces. In the second case, where the compression axis is tilted by $45^{\circ}$ with respect to $A 10$, the maximum of the resolved shear stress occurs in the quasiperiodic planes. The slipline pattern indeed indicates single-slip conditions with the quasiperiodic planes as slip planes. This conclusion is corroborated by the results of the Burgers vector analysis which yields directions inclined to the quasiperiodic planes in the first case and directions lying in these planes in the second.

The stress maximum of $200 \mathrm{MPa}$ and $183 \mathrm{MPa}$ at $780^{\circ} \mathrm{C}$ compares well with that found in single-quasicrystalline icosahedral $\mathrm{Al}_{70.5} \mathrm{Pd}_{21} \mathrm{Mn}_{8.5}$ for compression along twofold axes with the same strain rate (Feuerbacher et al. 1995, 1997). Note that the homologous temperatures are comparable since the two materials have very similar melting points. The stress exponent is about the same for both materials. For the activation volume we find $2 \mathrm{~nm}^{3}$ at $100 \mathrm{MPa}$ and $1 \mathrm{~nm}^{3}$ at $200 \mathrm{MPa}$. The respective values for $\mathrm{Al}_{70.2} \mathrm{Pd}_{21} \mathrm{Mn}_{8.5}$ amount to $1.2 \mathrm{~nm}^{3}$ and $0.6 \mathrm{~nm}^{3}$ and are thus of the same order. The activation volume is much larger than the 'atomic' volume defined by $b_{\|}^{3} \cong 6 \times 10^{-2} \mathrm{~nm}^{3}$, where $b_{\|}$is the modulus of the physical-space component of a typical Burgers vector in decagonal Al-Ni-Co (Yan et al. 1994). This indicates that dislocation motion is controlled by obstacles rather than by a diffusion mechanism where $V$ should be of the order of the atomic volume.

Concerning the nature of the rate-controlling processes, we note that quasicrystalline materials are intermetallic alloys whose mechanical strength is connected with their chemical order. In the X-ray structure analysis of decagonal Al-Ni-Co by Steurer et al. (1993), columnar clusters, parallel to the tenfold axis, formed by ten pentagonal antiprismatic columns surrounding a central column, are found to be the basic structural building elements. In the quasiperiodic plane, these form an interconnected network of close icosagonal rings of pentagonal and rectangular structure motifs. A basic length scale is defined by the diameter of about $3 \mathrm{~nm}$ of the planar basic structural unit of a columnar cluster.

For icosahedral $\mathrm{Al}_{70.5} \mathrm{Pd}_{21} \mathrm{Mn}_{8.5}$ quasicrystals a cluster-friction model has been set up (Feuerbacher et al. 1997). In this alloy, icosahedral three-shell Mackay-type clusters, as the basic structural units, are forming an interconnected three-dimensional network. The model starts from the assumption that these clusters are acting as high-strength intrinsic rate-controlling obstacles to dislocation motion. The phenomenon of deformation softening is explained by the introduction of phason defects, that is matching-rule violations representing structural and chemical disorder, caused by the motion of dislocations through this structure whereby the clusters lose their mechanical strength.

Our observation of deformation softening in the case of compression along an axis inclined with respect to $A 10$ suggests that, in decagonal $\mathrm{Al}_{71 \cdot 7} \mathrm{Ni}_{15 \cdot 4} \mathrm{Co}_{12 \cdot 9}$, a basically similar rate-controlling mechanism is acting, the intrinsic obstacles being provided by the columnar clusters. In analogy to the case of icosahedral quasicrystals, a continuous weakening of the structure takes place by the introduction of structural and chemical disorder in the wake of moving dislocations cutting through the columnar cluster arrangements. This holds true for both orientations of the compression axis and accounts for the observed independence of the activation volume on specimen orientation. 
In the case of the inclined compression axis, in which the specimen, according to the slip-line pattern, is oriented for single slip along the quasiperiodic planes, deformation softening is the dominating process. In contrast, in the case of compression along $A 10$, where the specimen is oriented for multiple slip, a second process has to be taken into account. As in conventional metal plasticity, the interaction of dislocations on different slip planes leads to a reduction in the density of mobile dislocations and of their effective velocity, that is to work hardening. Under the conditions used in our experiments, the effect of work hardening then prevails over that of deformation softening, leading to the observed increase of stress with strain.

\section{REFERENCES}

Basov, D. N., Timusk, T, Barakat, F., Greedan, J., and Grushio, B., 1994, Phys. Rev. Lett., $72,1937$.

Bresson, L., and Gratias, D., 1993, J. non-crystalline Solids, 153\&154, 486.

Courtney, T. H., 1990, Mechanical Behavior of Materials (New York: McGraw-Hill).

Feuerbacher, M., Baufeld, B., Rosenfeld, R., Bartsch, M., Hanke, G., Beyss, M., Wollgarten, M., Messerschmidt, U., and Urban, K., 1995, Phil. Mag. Lett., 71, 91.

Feuerbacher, M., Metz macher, C., Wollgarten, M., Baufeld, B., Bartsch, M., Messerschmid t, U., and Urban, K., 1997, Mater. Sci. Engng. A, 233, 103.

Grushio, B., and Urban, K., 1994, Phil. Mag. B, 70, 1063.

Kang, S. S., and Dubois, J. M., 1992, Phil. Mag. A, 66, 151.

Kocks, U. F., Argon, A. S., and Ashby, M. F., 1975, Progress in Material Science, Vol. 19 (Oxford: Pergamon Press).

Köster, U., Liu, W., Liebertz, H., and Michel, M., 1993, J. non-crystalline Solids, 153\& 154, 446.

Lin, S., Wang, X., Li, L., Zhang, D., He, L.X., and Kuo, K. H., 1990, Phys. Rev. B, 41, 9625.

Mishra, R. S., Singh, A. K., and Roy, T., 1993, Phil. Mag. Lett., 68, 225.

Shibuya, T., Нashimoto, T., and Takeuchi, S., 1990, J. Phys. Soc. Japan, 59, 1917.

Steurer, W, 1996, Physical Metallurgy, edited by R. W. Cahn and P. Haasen (Amsterdam: Elsevier), p. 371.

Steurer, W., Haibach, T., Zhang, B., Kek, S., and Lück, R., 1993 Acta crystallogr. B, 49, 661.

Takeuchi, S., Imanaga, H., and Shibuya, T., 1991, Jap, J. appl. Phys., 30, 561.

Wang, R., Yan, Y.F., and Kuo, K. H., 1993, J. non-Crystalline Solids, 153\&154, 103.

Wittmann, R., Urban, K., Schandl, M., and Hornbogen, E., 1991, J. Mater. Res., 6, 1165.

Wollgarten M., Bartsch, M., Messerschmidt, U., Feuerbacher, M., Rosenfeld, R., Beyss, M., and Urban, K., 1995, Phil. Mag. Lett., 71, 99.

Wollgarten, M., Beyss, M., Urban, K., Liebertz, H., and Köster, U., 1993, Phys. Rev. Lett., 71, 549.

Yan, Y., Wang, R., Gui, J., and Dai, M. X., 1993, Acta crystallogr. B, 49, 435.

Yan, Y., Zhang, Z., and Wang, R., 1994, Phil. Mag. Lett., 69, 123. 
